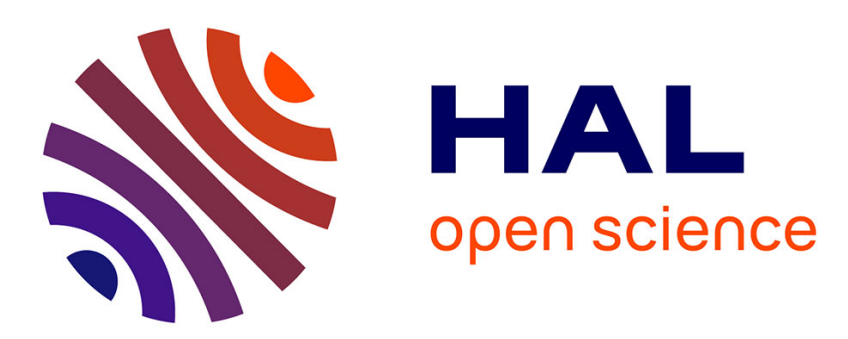

\title{
Réalisation d'un dispositif de mesure de la vitesse et de l'atténuation d'ondes ultrasonores dans des liquides sous pression
}

S. Ye, J. Alliez, B. Lagourette, H. Saint-Guirons, J. Arman, P. Xans

\section{- To cite this version:}

S. Ye, J. Alliez, B. Lagourette, H. Saint-Guirons, J. Arman, et al.. Réalisation d'un dispositif de mesure de la vitesse et de l'atténuation d'ondes ultrasonores dans des liquides sous pression. Revue de Physique Appliquée, 1990, 25 (6), pp.555-565. 10.1051/rphysap:01990002506055500 . jpa-00246218

\section{HAL Id: jpa-00246218 https://hal.science/jpa-00246218}

Submitted on 1 Jan 1990

HAL is a multi-disciplinary open access archive for the deposit and dissemination of scientific research documents, whether they are published or not. The documents may come from teaching and research institutions in France or abroad, or from public or private research centers.
L'archive ouverte pluridisciplinaire HAL, est destinée au dépôt et à la diffusion de documents scientifiques de niveau recherche, publiés ou non, émanant des établissements d'enseignement et de recherche français ou étrangers, des laboratoires publics ou privés. 


\title{
Réalisation d'un dispositif de mesure de la vitesse et de l'atténuation d'ondes ultrasonores dans des liquides sous pression
}

\author{
S. Ye, J. Alliez, B. Lagourette, H. Saint-Guirons, J. Arman et P. Xans \\ Laboratoire de Physique des Matériaux Industriels, Centre Universitaire de Recherche Scientifique, Avenue \\ de l'Université, 64000 Pau, France
}

(Reçu le 22 novembre 1989, révisé le 15 février 1990, accepté le 5 mars 1990)

\begin{abstract}
Résumé. - Cet article décrit en détail un dispositif de mesure de la vitesse et du coefficient d'atténuation d'ondes ultrasonores dans des liquides pouvant être portés à des pressions comprises entre 1 et 1000 bars. La cellule de mesure et les conditions spécifiques de son remplissage (lorsque les échantillons étudiés sont issus de fluides de gisement stockés dans des conditions de pression proches de celles du fond du puits) sont présentées plus particulièrement. Des comparaisons entre les valeurs expérimentales obtenues au moyen de ce dispositif et des valeurs relevées dans la littérature pour quelques corps purs (eau, décane, hexadécane) sont aussi rapportées.
\end{abstract}

\begin{abstract}
An apparatus for the measurement of ultrasonic velocity and attenuation in liquids at high pressures (up to $100 \mathrm{MPa}$ ) is described. Details of ultrasonic cell and transfer method of samples under pressure (e.g. fluids issue from natural petroleum reservoirs) are given. Our experimental results were compared with litterature values for some pure components (water, $n-C_{10}, n-C_{16}$ ).
\end{abstract}

\section{Introduction.}

La modélisation du comportement des fluides de gisement tant du point de vue des propriétés thermodynamiques que des propriétés d'écoulement présente un intérêt fondamental dans la perspective de l'exploitation des champs pétroliers. Elle repose sur la connaissance, basée soit sur des déterminations expérimentales directes soit sur des estimations déduites à partir d'équations d'état adaptées aux fluides, de certaines grandeurs physiques parmi lesquelles la masse volumique, les compressibilités isotherme et adiabatique, les chaleurs spécifiques, la viscosité dynamique,... Afin d'être exploitable en pratique, la description doit recouvrir intégralement les domaines de pression et de température rencontrés dans les gisements, aspect contraignant dans la mesure où la détermination expérimentale directe de certaines grandeurs n'est accessible que très difficilement ou avec des précisions faibles. C'est particulièrement le cas pour les expériences à mener en pression. Pour cette raison la détermination de la vitesse de propagation $U$ d'ultrasons dans des huiles pétrolières ou des mélanges types de corps hydrocarbonés semble d'un grand intérêt compte tenu des corrélations classiques entre cette grandeur et cer- tains paramètres thermodynamiques. Le haut degré de précision avec lequel la vitesse peut être connue permet en outre de remonter aux paramètres avec une précision très acceptable, souvent supérieure à celle d'une mesure thermodynamique directe (Van Itterbeek, 1965 [1]). Un autre avantage inhérent à la mise au point d'un dispositif de mesure de vitesse et d'atténuation d'ultrasons dans des fluides sous hautes pressions réside dans la possibilité de déceler assez commodément et avec une très bonne reproductibilité les changements d'état dans les fluides étudiés, notamment l'apparition du point de bulle ou des transitions liquide-liquide, ... De plus de telles mesures pourront être utilisées pour compléter des investigations menées sur les champs de production par diverses techniques sismique et sonique et de caractérisation des réservoirs. Enfin la connaissance de $U$ peut être reliée aux données $(P, V, T)$ du fluide et utilisée comme test de validité pour une étude critique comparative des équations d'état habituellement prises en compte dans l'industrie pétrolière.

Bien que la $v$ tesse du son dans divers liquides à la pression atmosphérique ait été mesurée par de nombreux chercheurs, les études en fonction de la pression sont beaucoup plus rares malgré l'intérêt présenté. Néanmoins un certain nombre de disposi- 
tifs de mesure de $U$ dans les fluides (liquides ou gaz) sous hautes pressions ont été mis au point parmi lesquels on peut citer ceux de Van Itterbeek (1965) [1] (appliqué à l'étude de plusieurs gaz liquifiés), de Bœlhouwer (1967) [2] (utilisé notamment sur des alcanes liquides), de Bobik (1978) [3], de Denielou et al. (1983) [4] (silicates fondus), de Makita et Takagi (1968) [5], de Dmitriev et Sokolov (1984) [6], de Kagramanyan et Badalyan (1978) [7], de Wang et al. (1988) [8] (huiles pétrolières), ...

Après avoir étudié la viscosité et la masse volumique sous hautes pressions de divers liquides proches ou issus d'huiles pétrolières et procédé à divers essais de représentation numérique (Ducoulombier et al. (1985) [9], Kanti et al. (1989) [10], Zhou et al. (1989) [11]) nous avons été amenés à nous intéresser à notre tour à l'évaluation de la vitesse du son dans ces fluides, pour différentes conditions de pression et de température. Nous nous proposons dans cet article de décrire le dispositif expérimental développé ainsi que ses caractéristiques techniques. Nous donnerons un aperçu de quelques résultats obtenus sur des liquides purs que nous avons comparés aux valeurs de la littérature.

\section{Principe général de la méthode de mesure de la vitesse et de l'atténuation.}

La méthode retenue, déjà expérimentée dans le laboratoire par J. Arman lors de l'étude, à la pression atmosphérique, de polymères fondus est celle de transmission d'impulsions ultrasonores à travers l'échantillon liquide. Nous opérons en utilisant deux transducteurs, l'un servant d'émetteur, l'autre de récepteur, disposés aux deux extrémités de la cellule de mesure schématisée sur la figure 3 et séparés de l'échantillon par deux barreaux cylindriques. Cette disposition présente le double avantage de pouvoir isoler les céramiques piézoélectriques du fluide sous pression et de les maintenir à une température constante proche de la température ambiante par le biais d'une circulation d'eau.

L'impulsion ultrasonore (de fréquence $5 \mathrm{MHz}$ ) est produite par le transducteur émetteur excité par un signal électrique de même fréquence. Après propagation à travers les barreaux et l'échantillon fluide, ce signal ainsi que les différents échos qui en résultent par suite des réflexions successives aux interfaces, sont détectés par le transducteur récepteur, convertis puis reproduits sur l'écran d'un oscilloscope.

Les temps de passage de l'onde ultrasonore dans les barreaux ayant été préalablement définis à la suite d'étalonnage il est possible d'identifier le trajet exact relatif à chacun des pics apparaissant sur l'oscilloscope.

Dans le cas de fluides fortement absorbants on peut déterminer la vitesse de propagation des ultra- sons par la mesure du temps séparant le signal de référence et le premier pic. Dans le cas de fluides moins absorbants, pour lesquels plusieurs échos sont visibles de manière significative, il est préférable, dans le but d'améliorer la précision, d'évaluer l'intervalle de temps séparant des échos non consécutifs.

L'évaluation directe du temps séparant deux pics sur l'écran de l'oscilloscope au moyen de la base de temps de cet appareil ne s'avère toutefois pas suffisamment précise. Pour cette raison nous avons utilisé la méthode dite "echo overlap method» décrite par May (1958) [12] et Papadakis (1964) [13] consistant à superposer les deux échos retenus pour la mesure, après les avoir isolés grâce à deux portes d'intensification, au moyen d'un balayage externe. De plus amples détails sont exposés dans l'article de J. Arman (1979) [14].

L'évaluation de l'atténuation de l'onde durant son parcours est une opération délicate à réaliser dans la mesure où les sources d'erreurs sont multiples (perte d'énergie due au couplage électromécanique, défaut de parallélisme éventuel des faces planes réfléchissantes en regard, perte par diffractions, ...) et peuvent fausser complètement les résultats. Pour s'affranchir de ces difficultés, le principe d'une méthode différentielle a été retenu; il consiste à suivre les variations de niveau d'un signal par comparaison à un signal interne de référence, une porte d'intensification étant alors placée sur le signal choisi. Ceci permet de suivre les variations relatives de l'atténuation.

\section{Description des éléments du dispositif expérimen- tal.}

3.1 PARTIE ÉLECTRONIQUE (ÉMISSION-DÉTECTION DES SIGNAUX). - Cette partie essentielle de l'appareillage d'ensemble est schématisée sur la figure 1 . Elle est constituée par les divers éléments ci-après :

1. Un générateur de basse fréquence (B.F.) modèle $\mathrm{n}^{\circ}$ 1210-C de General Radio Company (gamme couverte de $20 \mathrm{~Hz}$ à $500 \mathrm{KHz}$ ).

2. Un fréquencemètre (périodemètre), modèle FH 2521 de Schlumberger, indiquant la fréquence émise par le générateur B.F.

3. Un appareil à fonctions multiples modèle 122 A de Matec qui permet de diviser la fréquence du signal du générateur B.F. par un facteur 10, 100, 1000 . Ses signaux sont ensuite utilisés pour synchroniser le générateur d'impulsion H.F., le balayage horizontal de l'oscillographe et l'enregistreur automatique d'atténuation. Lorsque les échos ultrasonores sont visualisés sur l'oscilloscope il permet aussi, grâce à deux portes d'intensification dont la position et la largeur sont variables, de sélectionner sur l'écran deux signaux quelconques. 


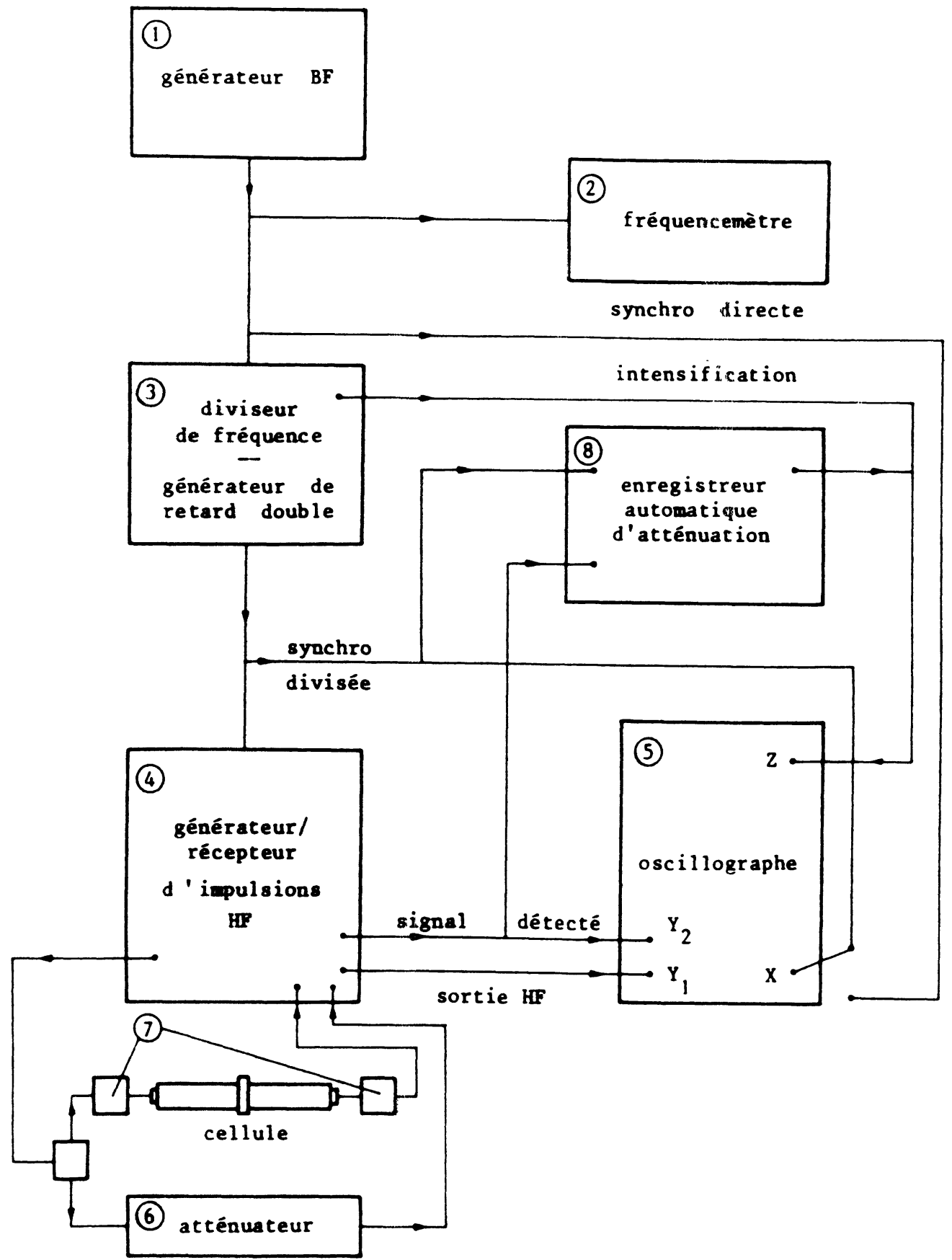

Fig. 1. - Schéma de la partie électronique du montage.

[Block diagram of the electronic equipment used.]

4. Un générateur, récepteur haute fréquence (H.F.) modèle 6600 de Matec muni du tiroir modèle 755 de Matec couvrant la bande de fréquence de 1 à $20 \mathrm{MHz}$. La durée des impulsions peut être choisie entre 0,5 et $100 \mu \mathrm{s}$; nous avons opéré avec des durées de $5 \mu \mathrm{s}$. Le taux de répétition ou intervalle de temps entre les impulsions dépend de la fréquence du générateur B.F. et du facteur de division utilisé ; dans nos expériences il est de l'ordre de $5 \mathrm{~ms}$. Ainsi la durée d'une impulsion étant le 1/1000 de l'intervalle séparant deux impulsions consécutives cette technique permet d'éviter l'inconvénient inhérent aux ondes continues à savoir l'échauffement et la dégradation des échantillons.

5. Un oscilloscope à double voie modèle OCT $388 \mathrm{~T}$ de Schlumberger, sur l'écran duquel sont visualisés les impulsions émises et les divers échos en résultant. Dans la méthode de Papadakis c'est la fréquence du générateur B.F. qui constitue la fréquence de balayage. Lorsque les deux signaux sélectionnés apparaissent exactement superposés sur 
l'écran cette fréquence du générateur B.F. est égale à l'inverse du temps séparant les deux signaux. De cette mesure du temps sera déduite la valeur de la vitesse $U$.

6. Un atténuateur calibré type 659 de Arenberg Ultrasonic Laboratory pouvant atténuer le signal direct de 1 à $122 \mathrm{~dB}$ par pas de $1 \mathrm{~dB}$.

7. Deux adaptateurs d'impédance destinés à accorder la sortie du générateur et l'entrée du récepteur $(50 \Omega)$ à l'impédance des transducteurs.

8. Enfin pour les mesures d'atténuation un enregistreur automatique d'atténuation modèle $2470 \mathrm{~A}$ de Matec sert à mesurer la différence de niveau entre deux signaux quelconques.

3.2 DisPositif D’ENSEMBLE. - La partie émissionréception des signaux ultrasonores que l'on vient de décrire est un élément du dispositif d'ensemble représenté sur la figure 2 qui comporte en outre :

- une pompe volumétrique à mercure bi-corps ; - une cellule tampon d'une capacité de $250 \mathrm{cc}$ réalisée en acier inoxydable, susceptible de supporter des pressions supérieures à 2 kbars, dont le rôle consiste à éviter l'introduction malencontreuse des échantillons liquides dans les corps de pompe;

- la cellule autoclave contenant le fluide à étudier ;

- enfin les périphériques servant à la régulation thermique ainsi que les capteurs de pression et température.

3.2.1. Cellule de mesure. - La cellule de mesure, représentée sur la figure 3 , est à barreaux intermédiaires entre l'échantillon et les transducteurs piézoélectriques. La partie autoclave en acier inoxydable (qualité APX4-Z8 CND) est composée d'un cylindre creux $\left(\varnothing_{\text {int }}=36 \mathrm{~mm}, \varnothing_{\text {ext }}=75 \mathrm{~mm}\right.$, longueur $=$ $168 \mathrm{~mm}$ ) constituant le corps de la cellule et de deux barreaux cylindriques, identiques entre eux et usinés avec le même acier $(\varnothing=32 \mathrm{~mm}$, longueur $=$ $50 \mathrm{~mm}$ ), centrés sur l'axe et fixés à chaque extrémité du corps par une culasse dont le serrage assure l'étanchéité par l'intermédiaire d'un joint torique métallique revêtu de téflon. La distance effective entre les barreaux, égale à $90,3 \mathrm{~mm}$ dans les conditions normales, a été choisie de façon à obtenir une précision convenable (de l'ordre de $\pm 0,5 \mathrm{~m} / \mathrm{s}$ ) sur la vitesse des ultrasons dans le fluide étudié, ce dernier baignant totalement leurs extrémités à l'intérieur de la cellule. A l'extrémité extérieure de chacun des barreaux est placé un transducteur piézoélectrique (de diamètre $\varnothing=15 \mathrm{~mm}$ ), de fréquence d'oscillation $5 \mathrm{MHz}$ (marque : Quartz et Silice), l'un servant d'émetteur, l'autre de récepteur. Ils sont solidaires des barreaux par l'intermédiaire d'un blocage mécanique et d'un liant visqueux assurant l'adhérence et surtout une bonne adaptation d'impédance acoustique. Pour ne pas modifier cette dernière lors

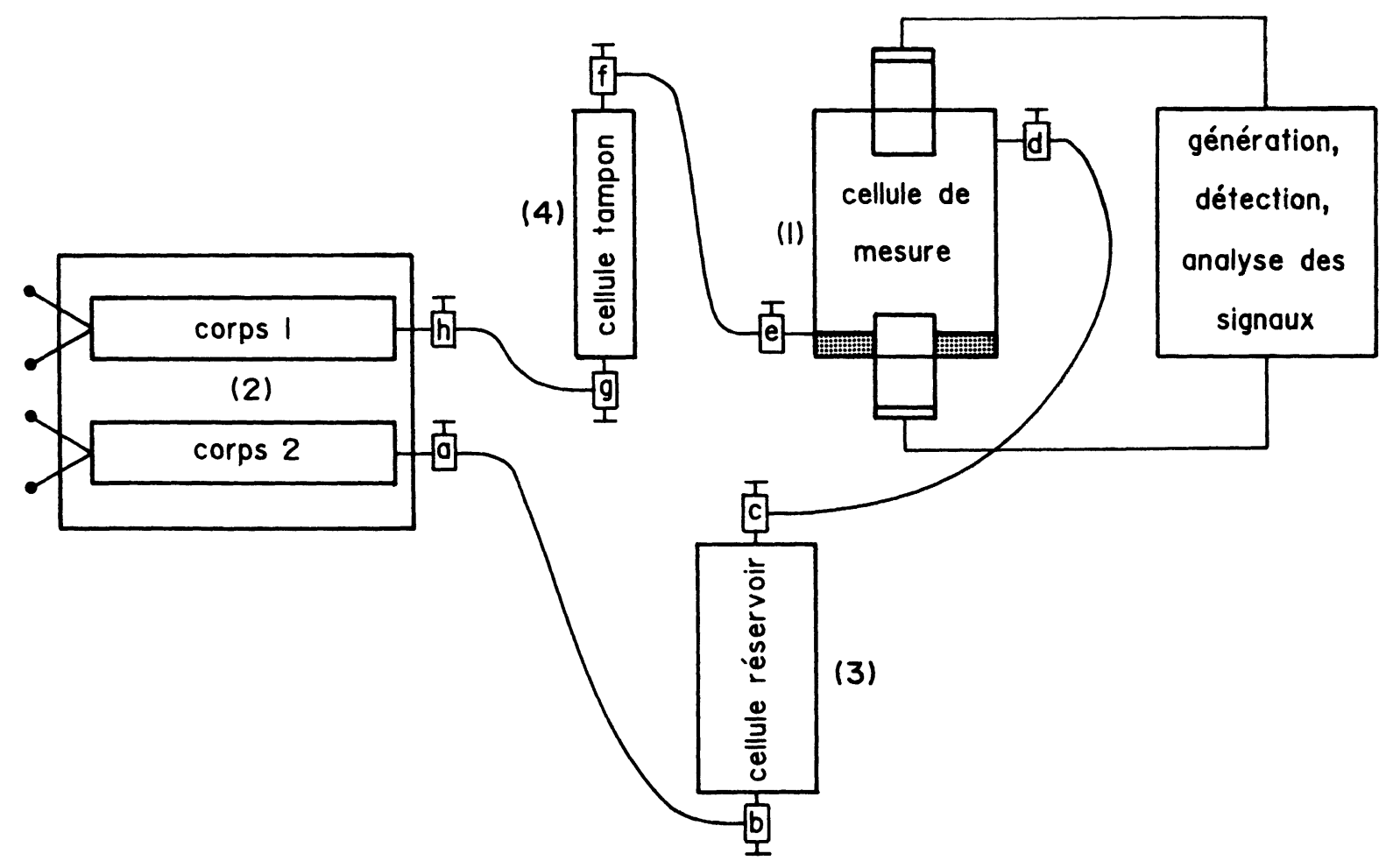

Fig. 2. - Schéma des divers éléments du dispositif.

[Functional diagram of the whole set-up.] 


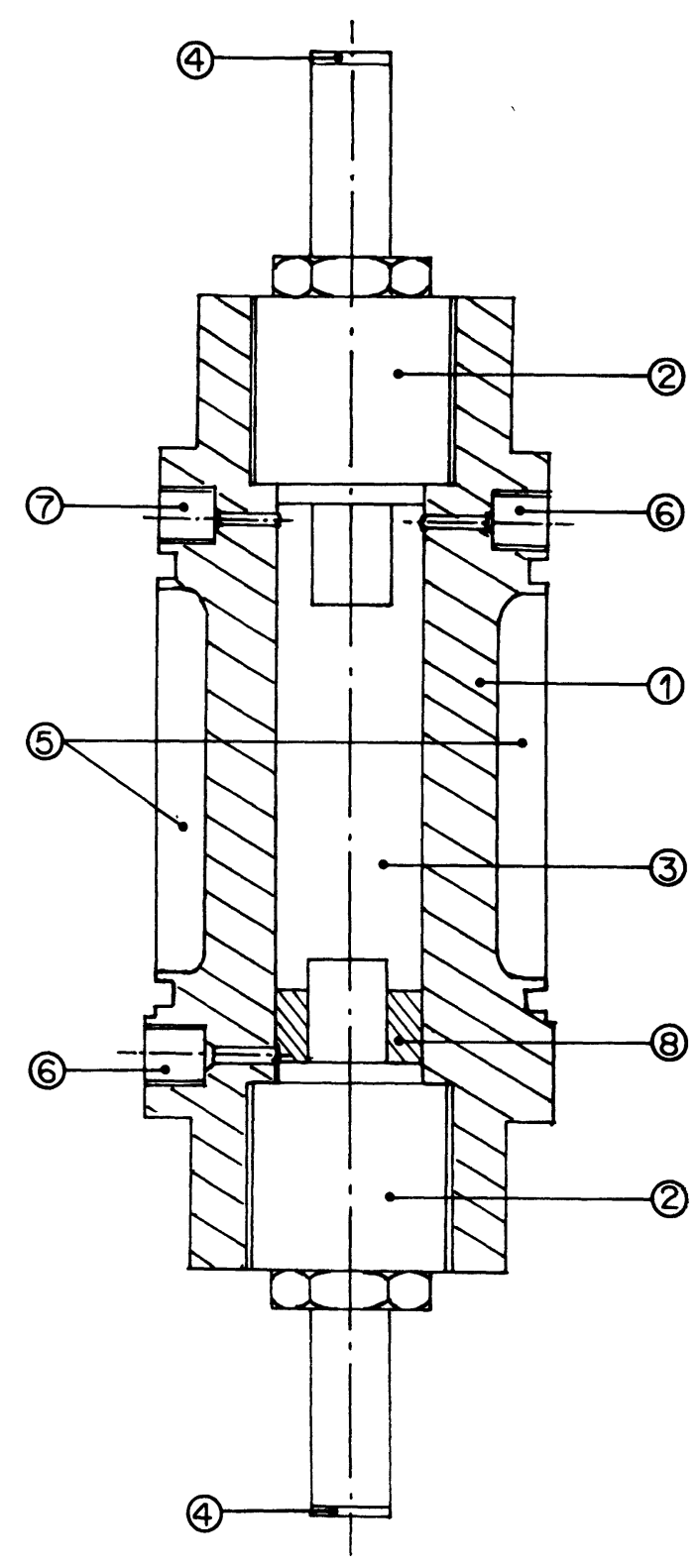

Fig. 3. - Représentation de la cellule de mesure.

[Measuring cell.]

d'études à différentes températures, les transducteurs sont entourés de radiateurs à eau qui assurent leur maintien à température constante, proche de la température ambiante. Afin d'accéder aux deux extrémités du volume interne utile, deux ouvertures ont été percées dans la partie latérale de la cellule, symétriquement par rapport à son centre ; elles sont fermées au moyen de deux vannes. Ceci permet, en disposant la cellule en position inclinée d'évacuer totalement le fluide échantillon après son étude. Un double passage électrique isolé et étanche a été réalisé suivant une technique décrite par ailleurs (Malfait et Jérôme (1969) [15], modifiée par SaintGuirons (1985) [16]); il permet le repérage de la température au sein même de l'échantillon à l'aide d'un couple thermo-électrique.

La partie autoclave est entourée d'une couche cylindrique en téflon, d'épaisseur $e=20 \mathrm{~mm}$, destinée à l'isolation thermique. La régulation en température est assurée par une circulation d'huile dans un espace annulaire ménagé entre le corps principal de la cellule et le cylindre en téflon. Ce circuit d'huile est relié à un bain thermostatique Lauda MGW 66 permettant une régulation à $\pm 0,05^{\circ} \mathrm{C}$ lors d'études isothermes. Le passage d'une température d'étude à une autre est accéléré par l'intermédiaire de deux colliers chauffants placés sur le corps même de la cellule et pouvant délivrer une puissance totale allant jusqu'à $600 \mathrm{~W}$. Le domaine de régulation thermique accessible avec ce matériel s'étend de $20^{\circ} \mathrm{C}$ à $150^{\circ} \mathrm{C}$.

3.2.2 Pompe volumétrique bi-corps à mercure. - La mise en pression des échantillons étudiés puis leur maintien à la pression voulue sont obtenus à l'aide d'une pompe volumétrique Ruska modèle 2218 TQM 580 à mercure, comportant un double corps. Le modèle utilisé contient $500 \mathrm{~cm}^{3}$ de mercure et permet d'accéder à des pressions de l'ordre de 1000 bars au maximum.

Chaque corps de pompe est équipé d'une échelle graduée, elle-même munie d'un vernier de subdivision, servant au repérage de la position instantanée des pistons internes et par voie de conséquence à la détermination des volumes de mercure déplacés. La précision de cette évaluation est de $0,01 \mathrm{~cm}^{3}$. Ce dispositif autorise aussi le transfert des fluides, à pression constante, entre les réservoirs de stockage et la cellule de mesure.

\section{Technique de remplissage de la cellule de mesure.}

Deux situations distinctes peuvent se présenter lors de la phase de remplissage selon que l'échantillon liquide à étudier se trouve à la pression atmosphérique ou bien à une pression supérieure à celle-ci.

4.1 CAS DES FLUIDES LIQUIDES À PRESSION AMBIANTE. - Pour de tels fluides (majorité des alcanes purs et de leurs mélanges), le remplissage de la cellule ne pose aucune difficulté particulière et s'effectue sans intervention des éléments périphériques. La cellule de mesure est totalement déconnectée du montage schématisé sur la figure 2. Le liquide est alors introduit par l'entrée inférieure (e) de la cellule et pénètre dans celle-ci sous l'effet des forces de gravité, la vanne supérieure (d) étant ouverte.

4.2 CAS DES fluides SOUS PRESSION. - Ce cas concerne deux catégories de fluides essentiellement :

- des mélanges de synthèse dont certains constituants se trouvent à l'état gazeux à la pression atmosphérique et aux températures proches de la 
température ambiante (par exemple binaire $\left.\mathrm{CO}_{2}+\mathrm{n}-\mathrm{C}_{16}\right)$;

- des échantillons de fluides pétroliers prélevés et conservés dans des conditions de pression proches de celles du fond du puits.

Tous ces fluides sont conservés avant étude dans des réservoirs pressurisés, d'une capacité de l'ordre de $600 \mathrm{~cm}^{3}$, pouvant supporter des pressions supérieures à 2000 bars.

Le transfert dans la cellule de mesure doit obligatoirement, de ce fait, être effectué sous pression (généralement comprise entre 50 et 300 bars selon les produits).

L'ensemble, constitué de la cellule de mesure (1), de la cellule tampon (4) et des capillaires métalliques de liaison assurant le raccordement de ces éléments au corps $n^{\circ} 1$ de la pompe, est parfaitement rempli de mercure (sans piégeage de bulles d'air) puis porté à la même pression que celle, connue, du réservoir (3) de l'échantillon. Le transfert peut désormais être effectué (vanne (c) ouverte) en utilisant simultanément les 2 corps de pompe disponibles en position de fonctionnement synchronisé. Cette technique présente l'avantage de transférer le fluide à pression constante, celle du réservoir de stockage. Les corps de pompe étant munis d'échelles de repérage de la position des pistons internes, on peut contrôler à tout instant le volume utile de fluide refoulé du réservoir (3) vers la cellule (1) et poursuivre ainsi l'opération jusqu'à atteindre le volume nécessaire à la manipulation envisagée.

\section{Homogénéisation des échantillons préliminaire- ment à leur transfert.}

Pour certains mélanges liquide-liquide ou liquidegaz susceptibles de présenter des phases hétérogènes, il est nécessaire d'homogénéiser l'échantillon préliminairement à son transfert de façon à introduire dans la cellule de mesure un échantillon dont les concentrations en divers constituants soient exactement représentatives du mélange stocké. Pour y parvenir on comprime l'échantillon jusqu'à une pression suffisamment élevée pour que le fluide se trouve dans le domaine monophasique liquide. La simple compression s'avérant souvent insuffisante pour rendre l'échantillon parfaitement homogène au plan de la distribution des concentrations, une agitation complémentaire est donc indispensable. Un support rotatif du réservoir permet de la réaliser avec une fréquence de 20 oscillations/min et une amplitude angulaire de $\theta_{0}=170^{\circ}$, le réservoir étant préalablement déconnecté de la pompe. Lorsqu'on rebranche le réservoir (3) au corps $n^{\circ} 2$ de la pompe, une chute de pression est en général observée. La même procédure est alors répétée jusqu'à ce que la modification de pression consécutive à l'agitation devienne négligeable.

\section{Etalonnage et vérifications.}

La mesure de la vitesse de propagation dans le fluide à étudier

$$
\left[U(P, T)=\frac{(n+1) L(P, T)}{\Delta t}\right]
$$

repose sur la détermination expérimentale du temps de passage $\Delta t$ (à 0,01 microseconde près) d'un train d'onde ultrasonore entre les deux extrémités des barreaux (ou après $n$ réflexions sur leurs extrémités). La distance $L(P, T)$ séparant les faces en regard des barreaux doit être connue le plus exactement possible, d'où la nécessité de prendre en compte des effets correctifs en température et pression, effets qui ont été précisés à l'aide d'un étalonnage préalable. Ce dernier a été mené avec du benzène (de marque Fluka, qualité puriss dont le degré de pureté dépasse $99,5 \%$ ), les valeurs de la vitesse ultrasonique dans le benzène prises pour référence étant celles de Bobik (1978) [3].

L'étalonnage est conduit en vue de l'évaluation de la vitesse de propagation au sein du fluide. L'existence de gradients thermiques dans les barreaux métalliques (notamment aux plus hautes températures d'étude) n'entache pas à notre sens la mesure expérimentale de $U$. Cette dernière résulte d'une comparaison à une autre mesure réalisée sur un fluide de référence dans des conditions identiques en pression et température. Or les délais que nous imposons entre l'établissement d'une situation $(P, T)$ donnée et la mesure expérimentale sont suffisamment importants pour qu'un régime stationnaire soit bien établi et pour espérer une reproductibilité fidèle des gradients en question. En ce qui concerne la détermination du coefficient relatif d'atténuation, la mesure implique la comparaison, à une même température et pour différentes pressions, $\mathrm{du}$ comportement du fluide à l'égard des ondes ultrasonores. Or le passage d'une pression à une autre n'affecte qu'imperceptiblement les gradients thermiques internes aux barreaux. L'influence des gradients sur la détermination du coefficient est ainsi négligeable. Pour vérifier la validité de l'étalonnage réalisé, des déterminations complémentaires de vitesses ont été effectuées pour d'autres corps purs : l'eau ainsi que quelques alcanes linéaires $\left(\mathrm{n}-\mathrm{C}_{10}\right.$ et $\mathrm{n}-\mathrm{C}_{16}$ notamment).

Avec l'eau (permutée puis bi-distillée), la vérification a été faite dans les intervalles $0-475$ bars et $15-$ $70{ }^{\circ} \mathrm{C}$ par comparaison aux valeurs de Wilson (1959) [17]. Le tableau I contient les divers résultats obtenus, les écarts observés n'excédant pas 0,6\%o.

Avec le n-décane et le n-hexadécane (marque Fluka, qualité puriss), les domaines de vérification ont été étendus jusqu'à 700 bars pour la pression et jusqu'à $120^{\circ} \mathrm{C}$ pour la température de manière à couvrir le domaine expérimental habituellement concerné lors des études de fluides de gisement (constitués majoritairement d'alcanes). 
Tableau I. - Vitesses des ultrasons dans l'eau à différentes pressions et températures. Comparaison de nos résultats avec les valeurs obtenues à partir de la formule de Wilson à 20 constantes (1959) [17].

[Ultrasonic velocities in water at given pressures $P$ and temperatures $T$. Comparison of ours results with Wilson's data [17] (from his equation with 20 coefficients, 1959).]

\begin{tabular}{|c|c|c|c|c|c|c|c|c|c|c|}
\hline \multirow{2}{*}{$\mathrm{P}$ (bar) $T\left({ }^{\circ} \mathrm{C}\right)$} & \multicolumn{2}{|c|}{16,5} & \multicolumn{2}{|c|}{31} & \multicolumn{2}{|c|}{41} & \multicolumn{2}{|c|}{56} & \multicolumn{2}{|c|}{69,5} \\
\hline & $U_{\text {exp }}$ & $U_{\text {Wilson }}$ & $U_{\exp }$ & $U_{\text {Wilson }}$ & $U_{\text {exp }}$ & $U_{\text {Wilson }}$ & $U_{\text {exp }}$ & $U_{\text {Wilson }}$ & $U_{\text {exp }}$ & $U_{\text {Wilson }}$ \\
\hline 50 & 1479,1 & 1479,6 & 1520,7 & 1520,2 & 1539,0 & 1539,4 & 1556,9 & 1557,3 & 1564,5 & 1564,2 \\
\hline 100 & 1487,4 & 1487,7 & 1529,0 & 1528,6 & 1547,8 & 1548,0 & 1565,8 & 1566,3 & 1573,4 & 1573,6 \\
\hline 150 & 1495,8 & 1495,9 & 1537,3 & 1537,0 & 1556,3 & 1556,5 & 1574,7 & 1575,2 & 1583,3 & 1582,9 \\
\hline 200 & 1504,3 & 1504,1 & 1545,9 & 1545,3 & 1564,9 & 1565,1 & 1583,7 & 1584,0 & 1592,4 & 1592,1 \\
\hline 250 & 1512,1 & 1512,3 & 1554,1 & 1553,7 & 1573,5 & 1573,5 & 1592,4 & 1592,8 & 1601,2 & 1601,2 \\
\hline 300 & 1518,2 & 1520,6 & 1562,0 & 1562,0 & 1581,9 & 1582,0 & 1601,3 & 1601,5 & 1610,5 & 1610,2 \\
\hline 350 & 1528,7 & 1528,8 & 1570,3 & 1570,3 & 1590,5 & 1590,4 & 1610,2 & 1610,1 & 1619,2 & 1619,1 \\
\hline 400 & 1537,0 & 1537,1 & 1578,9 & 1578,6 & 1599,0 & 1598,7 & 1618,4 & 1618,7 & 1628,0 & 1627,9 \\
\hline 436 & & & & & & & & & 1634,4 & 1634,2 \\
\hline 438 & 1543,6 & 1543,4 & & & & & & & & \\
\hline 450 & & & & & 1607,5 & 1607,1 & & & & \\
\hline 462 & & & & & 1609,4 & 1609,0 & & & & \\
\hline 472 & & & 1589,9 & 1590,5 & & & & & & \\
\hline
\end{tabular}

Les valeurs des vitesses mesurées ont été comparées aux valeurs de la littérature (pour le $\mathrm{n}-\mathrm{C}_{10}$ à celles de Badalyan et al. (1970) [18], pour le $\mathrm{n}-\mathrm{C}_{16}$ à celles de Bœlhouwer (1967)). Les tableaux II et III résument ces divers résultats. En valeur relative, les écarts maximum relevés sont de $0,2 \%$. A titre d'exemple nous avons représenté sur les figures 4 et 5 l'ensemble de nos points expérimentaux et ceux des auteurs précédents relatifs au domaine de vérification du $n-C_{16}$ et du $n-C_{10}$ respectivement.

$\mathrm{Au}$ vu de ces diverses comparaisons, nous estimons que la cellule mise au point permet d'évaluer

Tableau II. - Vitesses des ultrasons dans le $\mathrm{n}^{-} \mathrm{C}_{10}$ à différentes pressions et températures. Comparaison de nos résultats avec les données de Badalyan (1970) [18].

[Ultrasonic velocities in $\mathrm{n}-\mathrm{C}_{10}$ at given pressures $P$ and temperatures $T$. Comparaison of our results with Badalyan's data (1970) [18].]

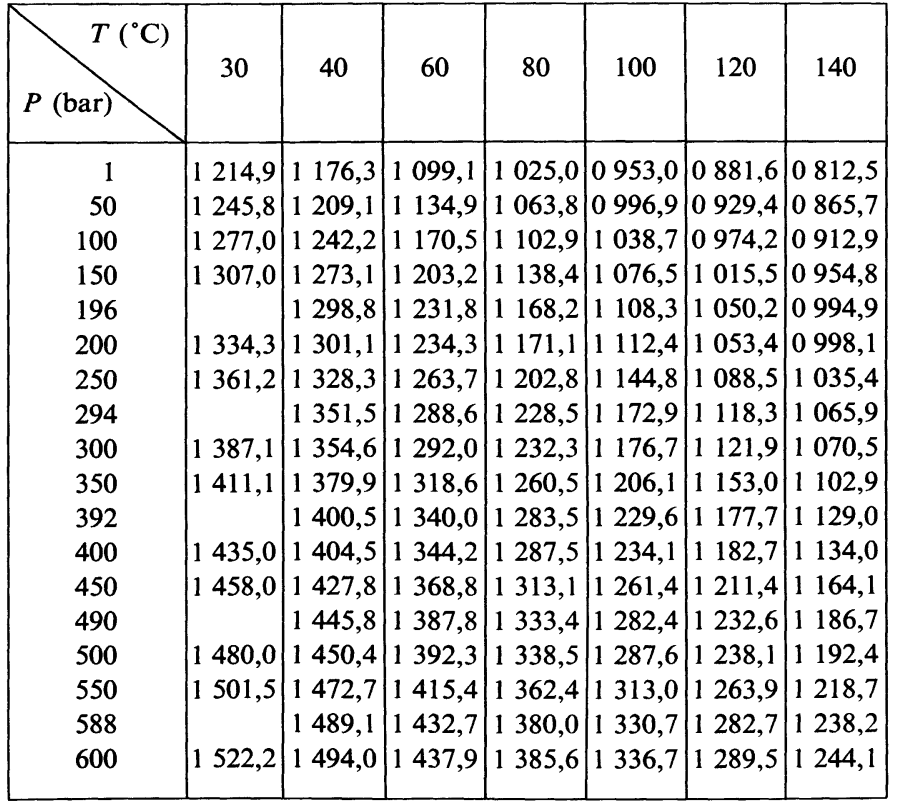

Nos mesures

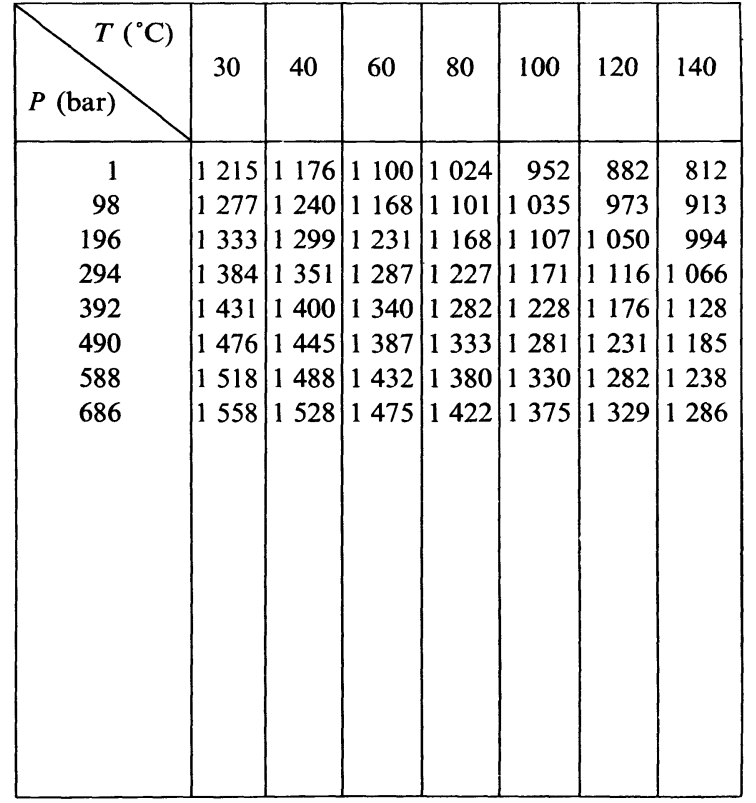

Mesures de Badalyan et al. 
Tableau III. - Vitesses des ultrasons dans le $\mathrm{n}-\mathrm{C}_{16}$ à différentes pressions et températures. Comparaison de nos résultats avec les données de Balhouwer (1967) [2].

[Ultrasonic velocities in $\mathrm{n}-\mathrm{C}_{16}$ at given pressures $P$ and temperatures $T$. Comparison of our results with Bœlhouwer's data (1967) [2].]

\begin{tabular}{|c|c|c|c|c|c|c|}
\hline$T\left({ }^{\circ} \mathrm{C}\right)$ & 30 & 40 & 60 & 80 & 100 & 120 \\
\hline 1 & 1320,6 & 1283,0 & 1212,1 & 1141,9 & 1075,1 & 1009,4 \\
\hline 50 & 1347,2 & 1311,9 & 1242,5 & 1174,8 & 1110,2 & 1049,4 \\
\hline 100 & 1376,6 & 1339,8 & 1272,6 & 1207,3 & 1147,1 & 1087,0 \\
\hline 150 & 1402,8 & 1366,4 & 1300,7 & 1237,7 & 1179,2 & 1122,0 \\
\hline 200 & 1424,1 & 1391,0 & 1327,4 & 1266,2 & 1210,0 & 1154,5 \\
\hline 250 & 1448,8 & 1416,0 & 1353,6 & 1293,9 & 1240,0 & 1185,1 \\
\hline 300 & 1472,3 & 1440,4 & 1379,1 & 1320,5 & 1267,7 & 1214,4 \\
\hline 350 & 1495,2 & 1463,5 & 1402,8 & 1345,6 & 1294,3 & 1242,3 \\
\hline 400 & 1516,4 & 1485,3 & 1425,6 & 1369,7 & 1319,5 & 1267,4 \\
\hline 450 & & 1507,9 & 1448,9 & 1393,2 & 1344,5 & 1293,9 \\
\hline 500 & & 1528,6 & 1470,8 & 1415,8 & 1368,4 & 1318,3 \\
\hline 550 & & & 1492,1 & 1438,0 & 1391,4 & 1342,2 \\
\hline 600 & & & 1512,4 & 1459,7 & 1413,7 & 1365,7 \\
\hline 650 & & & 1531,4 & 1480,6 & 1435,6 & 1388,8 \\
\hline 700 & & & & & 1457,4 & 1410,6 \\
\hline
\end{tabular}

Nos mesures

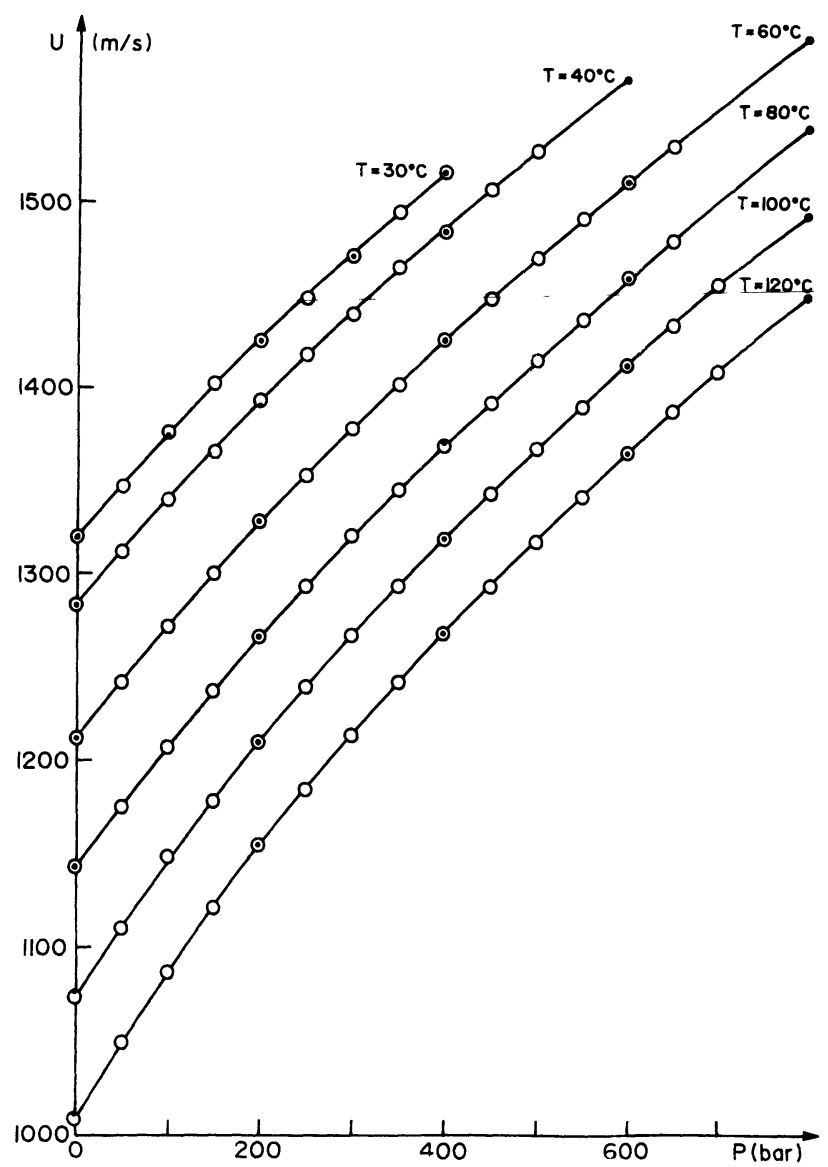

Fig. 4. - Courbe représentative des variations de $U$ en fonction de la pression, à température fixe, pour le n$\mathrm{C}_{16}$ : (O) points correspondant à nos mesures, ( $\bullet$ points de Bœlhouwer [2].

[Variations of sound velocity in $\mathrm{n}-\mathrm{C}_{16}$ as a function of pressure for different temperatures: $(O)$ our measured values, (•) Bœlhouwer data [2].]

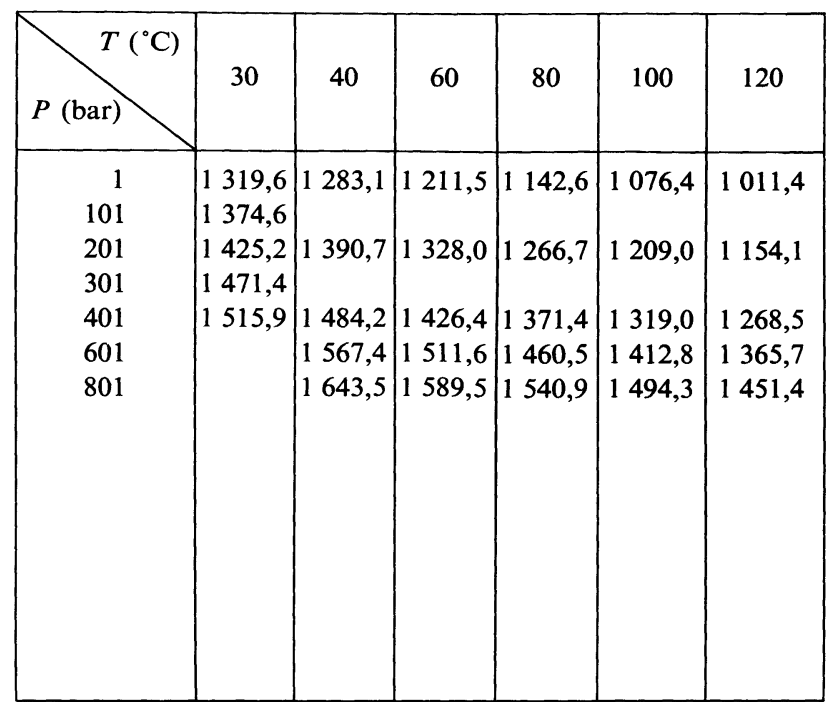

Mesures de Bœlhouwer

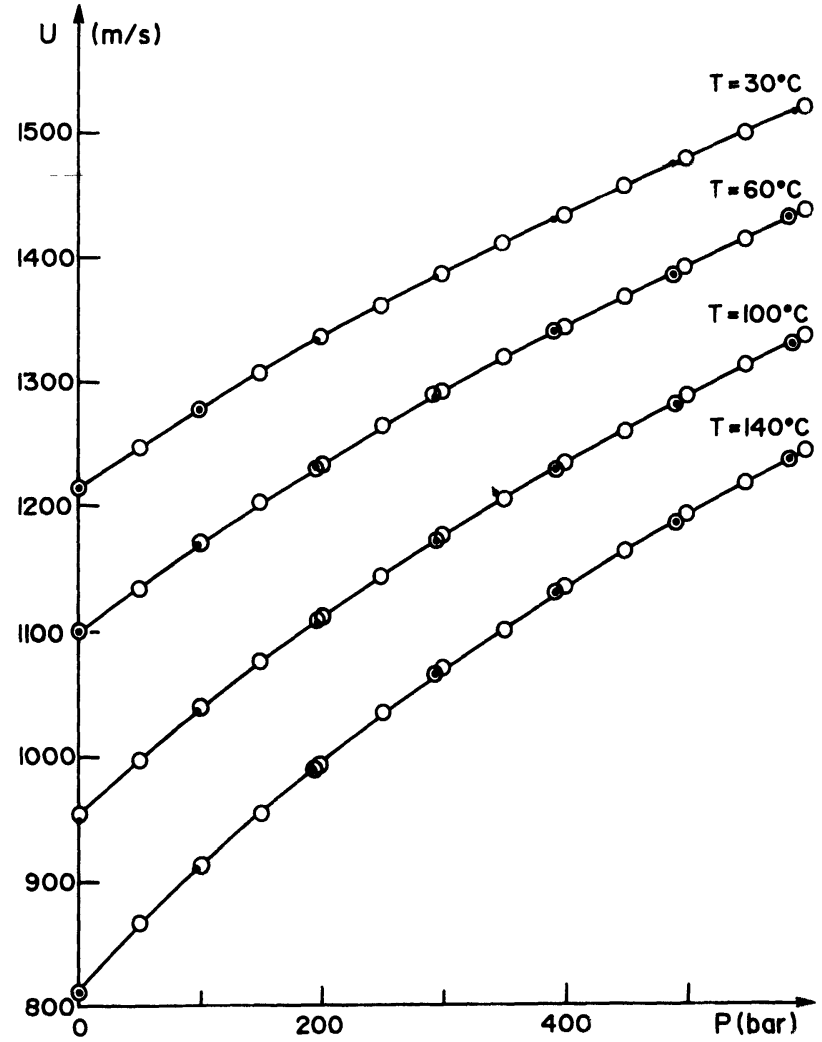

Fig. 5. - Courbe représentative des variations de $U$ en fonction de la pression, à température fixe, pour le n$\mathrm{C}_{10}$ : (O) points correspondant à nos mesure, (•) points de Badalyan et al. [18].

[Variations of sound velocity in $\mathrm{n}-\mathrm{C}_{10}$ as a function of pressure for different temperatures: (O) our measured values, (๑) Badalyan et al. data [18].] 
les vitesses de propagation des ondes ultrasonores sous pression avec une précision correcte, de l'ordre de grandeur de celle des auteurs cités précédemment (environ $\pm 0,5 \mathrm{~m} / \mathrm{s}$ ).

Le dispositif décrit ci-dessus permet par ailleurs de suivre l'évolution de l'amplitude d'un écho particulier (associé à un trajet bien identifié, très souvent le trajet direct sans réflexion) à différentes pressions et pour une même température d'étude. Par comparaison de cette amplitude à celle d'un signal constant interne au générateur, l'enregistreur MATEC 2470 A fournit un coefficient relatif d'atténuation du fluide ( $r$ ) mais pas le coefficient absolu d'atténuation. Cette mesure, bien que moins prometteuse en information que celle de l'atténuation absolue (qui théoriquement est corrélée à quelques paramètres thermodynamiques spécifiques au fluide) est néanmoins utile dans la perspective de détecter les équilibres liquide-vapeur de fluides complexes tels les hydrocarbures ou produits pétroliers.
Sur les figures 6 et 7 nous avons représenté à titre d'exemple les variations du coefficient relatif d'atténuation du $\mathrm{n}-\mathrm{C}_{10}$ et du $\mathrm{n}-\mathrm{C}_{16}$ pour 3 isothermes.

Ces courbes révèlent une diminution du coefficient $r$ lorsque, à température fixe, la pression croît.

L'intérêt essentiel de la détermination de $r$ résulte de la très forte augmentation de ce paramètre lorsqu'apparaît la phase gazeuse dans l'échantillon, ce qui constitue un moyen fiable et efficace de préciser les conditions $(P, T)$ de la transition. En particulier cette technique permet de repérer avec précision le point de bulle dans les fluides de gisement (pour chaque isotherme exploré). Afin d'illustrer ceci, nous montrons sur la figure 8 les variations du coefficient $r$, mesuré à la température d'étude $T=20^{\circ} \mathrm{C}$, relatif au mélange binaire $\mathrm{CO}_{2}$ n- $\mathrm{C}_{16}$ de composition $x=0,90$ en $\mathrm{CO}_{2}$ ( $x$ désignant la fraction molaire).

La pression du point de bulle correspond à la valeur de $P$ pour laquelle $r$ subit une croissance

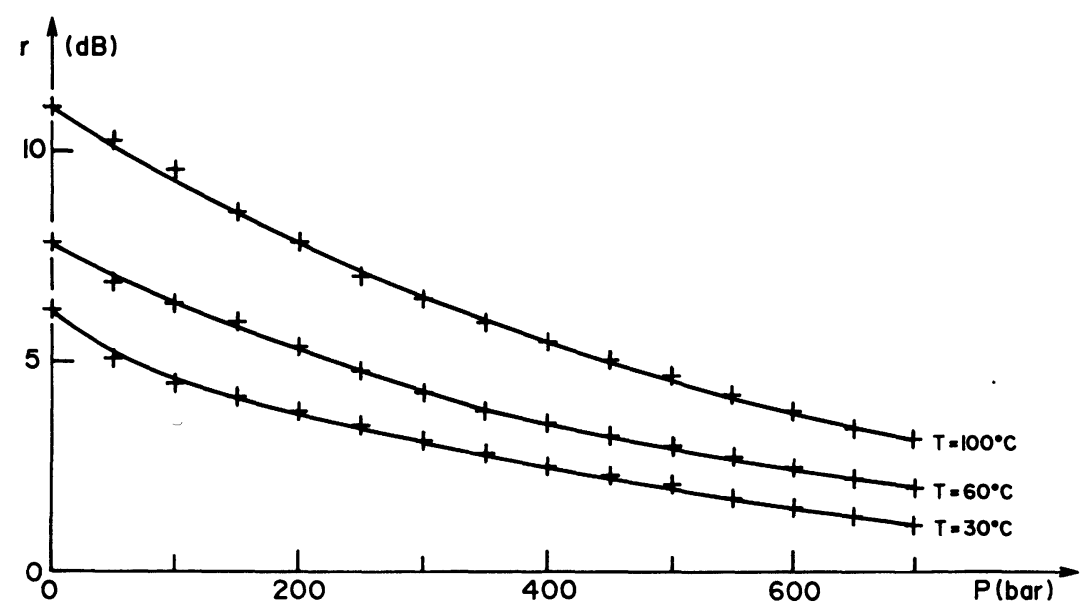

Fig. 6. - Variations du coefficient relatif d'atténuation des ultrasons, en fonction de la pression, dans le $n-C_{10}$. [Variations of relative ultrasonic attenuation coefficient versus pressure, in $n-C_{10}$.

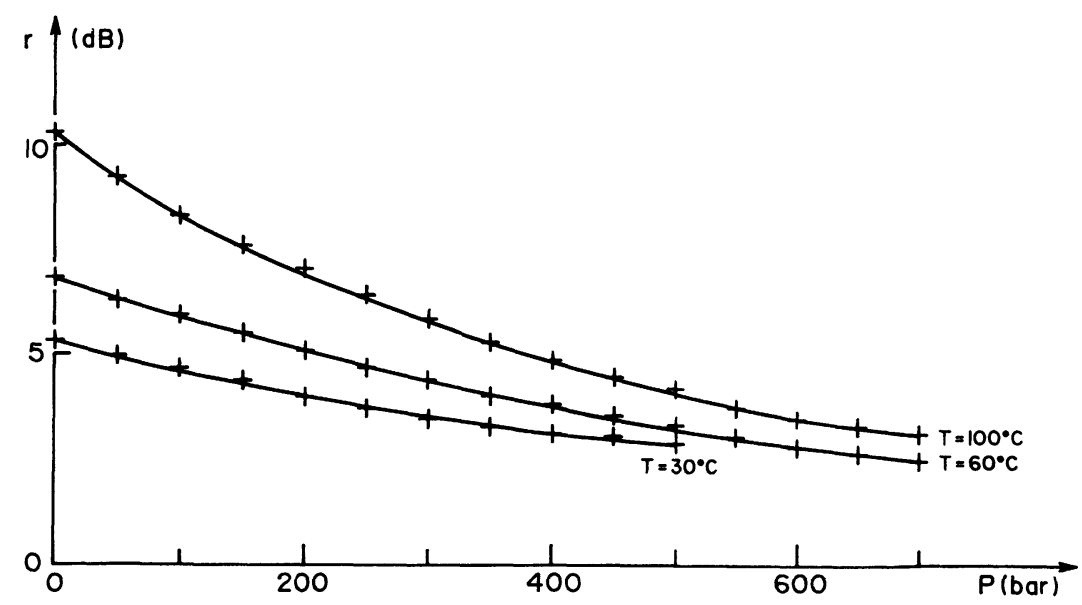

Fig. 7. - Variations du coefficient relatif d'atténuation des ultrasons, en fonction de la pression, dans le $n-C_{16}$. [Variations of relative ultrasonic attenuation coefficient versus pressure, in $\mathrm{n}-\mathrm{C}_{16}$. 


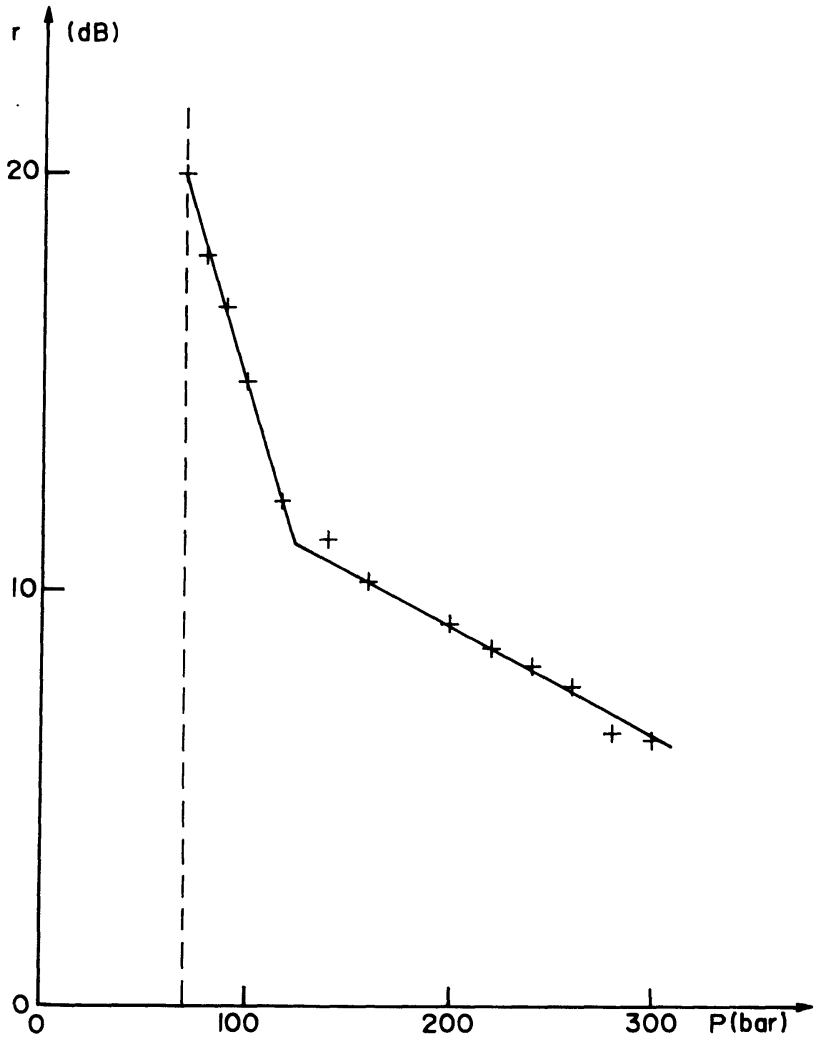

Fig. 8. - Représentation graphique du coefficient relatif d'atténuation (en fonction de la pression) dans le mélange binaire $\mathrm{CO}_{2}-\mathrm{n}-\mathrm{C}_{16}$ de fraction molaire $x=0,90$ en $\mathrm{CO}_{2}$, à la température d'étude $T=20^{\circ} \mathrm{C}$.

[Diagram of retative ultrasonic attenuation coefficient versus pressure, in $\mathrm{CO}_{2}+\mathrm{n}-\mathrm{C}_{16}$ mixture (molar ratio of $\mathrm{CO}_{2}, x=0.90$; experimental data at $T=20^{\circ} \mathrm{C}$ ). quasi infinie, la courbe isotherme étant décrite dans le sens des pressions décroissantes depuis une pression supérieure où le mélange est en état monophasique liquide jusqu'à une pression inférieure correspondant à celle du point de bulle. La première rupture de pente observable sur le diagramme peut être reliée à une transition liquide-liquide ainsi que le suggère le diagramme de phases du binaire, établi par Larsen et al. (1986) [19].

\section{Conclusion.}

L'utilisation de la pompe à double corps réduit au minimum les perturbations de l'état du fluide lors des opérations de transfert. La cellule ainsi que les autres parties du dispositif forment un ensemble robuste, précis, fiable, fortement spécialisé et dont l'intérêt est évident pour le secteur pétrolier. En pratique, ce dispositif sert à la détermination de diagrammes de phases par détection de transitions dans les fluides de gisement. Il est particulièrement bien adapté au repérage précis des points de bulle (à 1 bar près), l'apparition de ces derniers se manifestant par une brusque augmentation du facteur d'atténuation accompagnée d'une instabilité importante des signaux sur l'écran de l'oscilloscope. Les transitions liquide-liquide sont déterminées après examen des isothermes $U=f(P)$ sur lesquels elles impliquent des variations conséquentes de la courbure. Des résultats expérimentaux relatifs à différents fluides de gisement seront présentés dans un prochain article.

\section{Bibliographie}

[1] VAN ItTerbeek A., Physics of high pressures and the condensed phase (North-Holland Publishing Company-Amsterdam) Chap. 7 (1965) p. 297.

[2] Belhouwer J. W. M., Sound velocities in and adiabatic compressibilities of liquid alkanes at various temperatures and pressures, Physica 34 (1967) 484.

[3] BовIK M., Thermodynamic quantities for liquid benzene. 1 - Sound velocities between 283 and $463 \mathrm{~K}$ and up to $62 \mathrm{MPa}, J$. Chem. Thermodyn. 10 (1978) 1137.

[4] Denielou L., Petitet J. P., Tequi C., Syfosse G., Mesure de la vitesse du son sous pression dans les silicates liquides. Mise au point de la méthode sur un sel fondu, Bull. Minéral. 106 (1983) 139.

[5] Makita T. et TAKagi T., Ultrasonic velocity in and thermodynamic properties of benzene and carbon tetrachloride under pressures, Rev. Phys. Chem. Jpn 38 (1968) 41.

[6] DMitriev S. P. et Sokolov V. V., Measuring the parameters of liquid media at high pressures, Prib. Tekh. Eksp. USSR 27 (1984) 184.
[7] Kagramanyan L. S. et Badalyan A. L., Pulsed experimental arrangement for measurements of ultrasound velocity in liquids under pressure up to $2000 \mathrm{~atm}$, Izv. Akad. Nauk Arm. SSSR Fiz. 13 (1978) 478.

[8] Wang Z., Nur A. M., Stanford U., Batzle M. L., Acoustic velocities in Petroleum Oils. Society of Petroleum, Engineers (1988) 18163, 571.

[9] Ducoulombier D., Lazarre F., Saint-Guirons H., XANS P., Viscosimètre à corps chutant permettant de procéder à des mesures de viscosité de liquides sous hautes pressions, Rev. Phys. appl. 20 (1985) 735.

[10] Kanti M., Zhou H., Ye S., Boned C., LagouRetTe B., SaInt-Guirons H., Xans P., MoNTEL F., Viscosity of liquid hydrocarbons, mixtures and petroleum cuts, as a function of pressure and temperature, J. Phys. Chem. 93 (1989) 3860.

[11] Zhou H., Lagourette B., Alliez J., Xans P., MONTEL F., Development and application of Simha equation of state to calculation of the density of certain alkanes and their mixtures, $J$. Fluid Phase Equilibria 47 (1989) 153. 
[12] MAY J. E., Precise measurement of time delay, IRE Natl. Conv. Rec. 6 (1958) 134.

[13] PAPAdakis E. P., Ultrasonic attenuation and velocity in three transformation products in steel, $J$. Appl. Phys. 35 (1964) 1474.

[14] Arman J., Dispositif de mesure de la vitesse de propagation et du coefficient d'atténuation d'ultrasons dans les polymères, Acustica 43 (1979) 212.

[15] Malfait G. et JÉrome D., Techniques de hautes pressions à très basse température, Rev. Phys. Appl. 24 (1969) 467.

[16] SAINT-Guirons H., Réalisation et adaptation de systèmes thermiques différentiels pour des mesures sous haute pression. Application à l'étude du polymorphisme de corps organiques et à la caractérisation de mélanges de polymères, Thèse d'Etat, Pau (1985).

[17] WILSON W. D., Speed of sound in distilled water as a function of temperature and pressure, J. Acoust. Soc. Am. 31 (1959) 1067.

[18] Badalyan A. L., Otpushchennikov N. F., ShoyTOV U. S., The velocity of ultrasound in $n-$ Octane and n-Decane at high pressure, Izv. Akad. Nauk Arm. SSSR Fiz. 5 (1970) 448.

[19] Larsen L., Silva M. K., TAYlor M. A., Temperature dependence of $\mathrm{L}_{1}-\mathrm{L}_{2}-\mathrm{V}$ behavior in $\mathrm{CO}_{2}$ hydrocarbon systems, Paper Soc. Pet. Eng. 15399 presented at the 1986 SPE Annual Technical Conference \& Exhibition, New-Orleans (Oct. 5-8). 